\title{
INFLUENCE OF CHANNEL FADING CORRELATION ON PERFORMANCE OF DETECTOR ALGORITHMS FOR V-BLAST ARCHITECTURE
}

\author{
Bindu. $\mathrm{E}^{1}$ and B.V. R. Reddy ${ }^{2}$ \\ ${ }^{1}$ Research Scholar, GGSIP University, \\ Dept. of ECE, Amity School of Engg and Tech., New Delhi, India \\ ${ }^{2}$ Professor, USICT, \\ GGS Indraprastha University, New Delhi, India
}

\begin{abstract}
This paper analyzes the impact of fading correlation and cross polarization coupling on the error performance of $V$-BLAST MIMO system that employs detector algorithms like ZF, MMSE and $M L$ with ordering and successive cancellation. Simulation results show the BER performance of these detectors for different modulation schemes. It is observed that lesser the channel fading correlation and cross polarization coupling values better is the performance of these detectors. Study is extended to see the effect of transmit and receive antenna correlation on Ergodic MIMO capacity.
\end{abstract}

\section{KEYWORDS}

MIMO, V-BLAST, ZF, MMSE, ML, fading correlation, cross polarization coupling

\section{INTRODUCTION}

Multiple input multiple output (MIMO) system employs multiple transmit and receive antennas to provide high data rates without using additional bandwidth and power. In a rich scattering environment, the capacity increases linearly with the number of antennas without increasing the transmission power. This results in the possibility of transmitting at a higher data rate, by using spatial multiplexing (SM). Multiplexing gain is defined as the number of sub channels of the MIMO channel asymptotically viewed as a parallel channel. Due to this the corresponding transmission rate is multiplied by the number of transmit antennas, thereby increasing gain. With $N$ receive antennas for $M$ (where $M \leq N$ ) simultaneously transmitted data streams through independent flat Rayleigh channels, the $M$ streams of data can be separated by using a zeroforcing $(\mathrm{ZF})$ scheme, and $\mathrm{d}=(N-M+1)$ path diversity can be achieved by each of the $M$ streams [6]. But in most practical MIMO systems, independent channels are difficult to achieve. Layered Space Time (LST) codes can provide multiplexing gain and are spectrally efficient and hence suitable to realize SM. Channel fading and additive noise are main problems faced by MIMO 
LST receiver along with Multistream Interference (MSI). MSI arises due to the interaction of data streams with each other and this interference can be minimized through correct choice of detector algorithms [2]. Bell Lab Layered Space-Time (BLAST) detection algorithms combine linear (interference suppression) and nonlinear (serial cancellation) detection methods. Combination of Optimal Ordering with Successive Cancellation (SUC-OO) algorithms with Zero Forcing (ZF), Minimum Mean Square (MMSE) or Maximum Likelihood (ML) receivers can minimize Multistream interference [1].

For theoretical analysis, the channel is modeled as flat fading Rayleigh independent identically distributed (i.i.d) Gaussian variable. The antennas are assumed to provide independent channels for the received signals. However, in real propagation environment, the fades are not independent due to insufficient spacing between antenna elements. When the fades are correlated, the channel capacity decreases [9]. Presence of Line-of-Sight (LOS) component also makes the channel deviate from Rayleigh i.i.d. Another Theoretical assumption of identical polarization for both transmitting and receiving antenna also fails in practical scenario, due to Cross Polarization effect. This phenomenon can also lead to high bit error rate (BER)[2]. This aspect along with the impact of fading correlation on different detection schemes is studied in this paper.

Organization of the paper is as follows: Section II explains the basic MIMO channel model and its capacity along with the effect of fading correlation and cross polarization on the complex channel matrix H. In section III, VBLAST architecture with the receiver algorithms like ML, ZF and MMSE, with and without SUC-OO are discussed. Section IV discusses the simulation results followed by conclusion in section $\mathrm{V}$.

\section{MIMO CHANNEL MODE}

In MIMO system with $\mathrm{M}$ transmitting and $\mathrm{N}$ receiving antenna, the received signal vector $\boldsymbol{y}$ is

$$
\mathrm{y}=\mathrm{Hx}+\mathrm{n}
$$

where $\boldsymbol{H}$ is channel matrix of dimension $M \times N$ with complex coefficients for Rayleigh i.i.d flat fading channel and $\boldsymbol{n}$ is a wide sense stationary (WSS) noise vector with i.i.d. components and variance of $\sigma^{2}$.The advantage of using MIMO system is the increased channel capacity it can provide for the same signal conditions. The outage capacity of MIMO system having $M=N$ antennas and for channel unknown to the transmitter is given as [1]

$$
C=M \log _{2}(1+\rho) \quad \text { bits } / \mathrm{s} / \mathrm{Hz}
$$

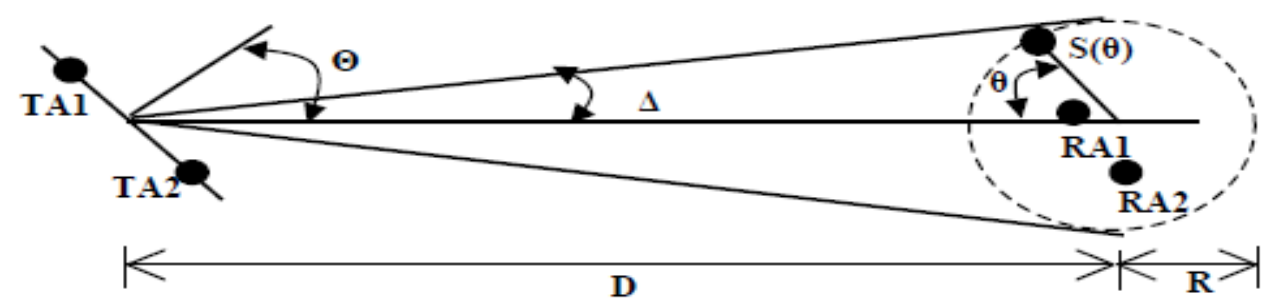

Fig. 1. One-ring model (TA -transmitting antenna, RA- receiving antenna, $S(\theta)$ - the scatterer at angle $\theta, \delta$ angle spread, D- the distance from object $\mathrm{X}$ to $\mathrm{Y}$ ) [9] 
where $\rho=E_{b} / N_{o}$. The capacity of an orthogonal MIMO channel is therefore $M$ times the scalar channel capacity.

Performance of MIMO system may deteriorate due to various factors, which influence the channel matrix $H$. Fading correlation due to closely spaced antenna and presence of LOS component due to poor scattering environment causes deviation of channel model from pure Rayleigh i.i.d. Cross polarization coupling between the antennas can also alter the channel matrix. Correlation problems arise because of the small separation distance between the antenna elements at the Base Station (BS). Fig.1 shows the "one-ring" model [9], which is used to determine spatial fading correlation of $H$. Due to the kind of geometry, all antennas will receive the same signal, giving rise to correlation between them. The normalized mutual correlation coefficient between any pair of antennas is [6]

$$
r_{i j}=E\left\{h_{i n} h_{j n}^{*}\right\}_{=} J_{o}\left(\left(\frac{2 \pi}{\lambda}\right) d^{R}(i, j)\right)
$$

where $(\cdot)^{*}$ denotes complex conjugate, $\mathrm{E}\{\cdot\}$ denotes statistical expectation, $\mathrm{J}_{0}(\cdot)$ is the zero order Bessel function of the first kind, $\lambda$ is the wavelength, and $d^{R}(i, j)$ is the displacement between receive antennas $i$ and $j$. Because of this, the elements of the channel matrix $H$ become [2]

$$
\mathrm{H}=\mathrm{R}_{\mathrm{r}}^{1 / 2} \mathrm{H}_{\omega} \mathrm{R}_{\mathrm{t}}^{1 / 2}
$$

where $R_{r}$ and $R_{t}$ are the receive and transmit covariance matrices (positive definite Hermitian matrices) of order $N \mathrm{x} N$ and $M \mathrm{x} M$ respectively. $H_{\omega}$ is a Rayleigh i.i.d complex Gaussian matrix with zero mean, unit variance entries. The correlation matrix $R$ with three receive antenna is $\mathrm{R}=\left[\begin{array}{ccc}1 & r_{2} & r_{13} \\ r_{21} & 1 & r_{23} \\ 3_{2} & r_{2} & \end{array}\right]$ where $r_{i j}$ is normalized mutual correlation coefficient between any pair of antenna, $r$ $=\mathrm{E}\left\{\mathrm{hh}^{\mathrm{H}}\right\}$, where $h$ is channel vector, $h=v e c[\mathrm{H}]$ [6]. Assuming receiver and transmitter matrix are of full rank, then at high SNR, the capacity can be approximated as

$$
C=\log _{2} \operatorname{det}\left(\frac{\rho}{M} \mathrm{H}_{\omega} \mathrm{H}_{\omega}^{\mathrm{H}}\right)+\log _{2} \operatorname{det}\left(\mathrm{R}_{\mathrm{r}}\right)+\log _{2} \operatorname{det}\left(\mathrm{R}_{\mathrm{t}}\right)
$$

As the equation shows, fading channel correlation reduces the outage channel capacity by $\left[\log _{2} \operatorname{det}\left(\mathrm{R}_{r}\right)+\log _{2} \operatorname{det}\left(\mathrm{R}_{t}\right)\right]$ bits/s/Hz.

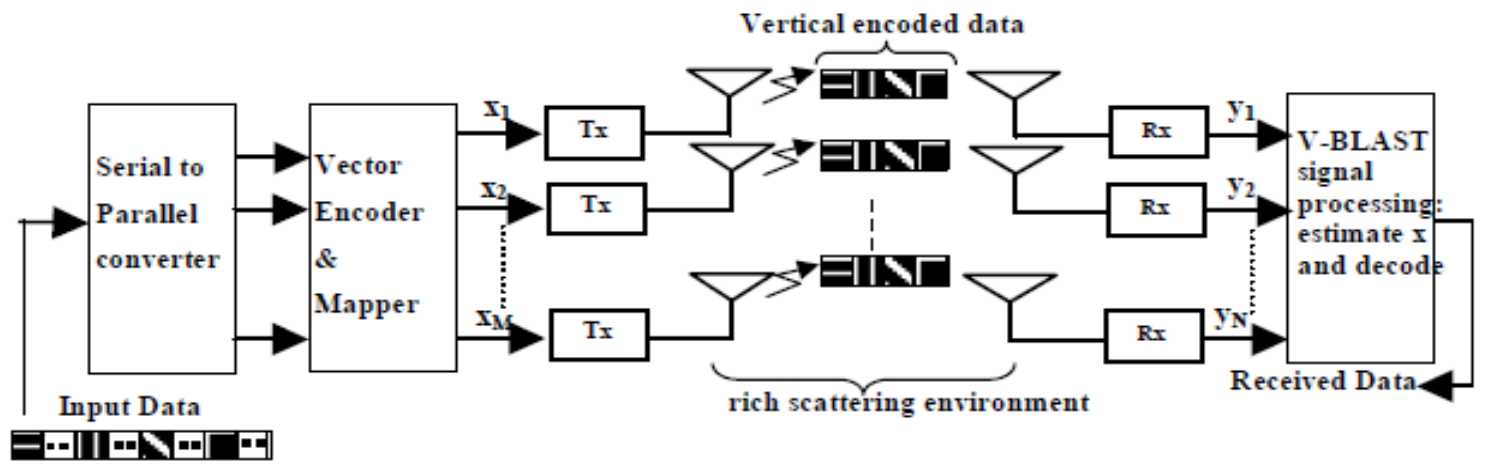

Fig.2 V-BLAST architecture [7] 
The loss caused by transmit and receive correlation is independent, the loss due to receive correlation is determined by the determinant of the receive correlation matrix and the numbers of receive antenna, and the loss caused by transmit correlated fading is determined by the transmit correlation matrix and the modulation scheme [9].

The use of antennas having orthogonal polarization at the transmitter and receiver leads to a gain and correlation imbalance between elements of $\mathrm{H}$. In reality, perfect polarization cannot be achieved, leading to cross polarization coupling [4]. The coupling factor $\alpha(0 \leq \alpha \leq 1)$ gives a measure of how well the antenna discriminates the polarization from other antenna. Assuming a Rayleigh i.i.d channel, the channel $\mathrm{H}$ with cross-polarized antenna can be modeled as [2]

$$
H=\beta \Theta\left(\mathrm{R}_{\mathrm{r}}^{1 / 2} \mathrm{H}_{\omega} \mathrm{R}_{\mathrm{t}}^{1 / 2}\right) \text { where } \beta=\left[\begin{array}{cc}
1 & \sqrt{\alpha} \\
\sqrt{\alpha} & 1
\end{array}\right]
$$

Where $\odot$ is Hadamard product. The capacity for a $2 \times 2$ system in non-scattering environment is given as

$$
C_{\alpha=0}=2 \log _{2}(1+\rho / 2) \text { and } C_{\alpha=1}=\log _{2}(1+2 \rho)
$$

\section{LAYERED SPACE TIME RECEIVERS}

The Vertical BLAST (V-BLAST) architecture is indicated in fig.2, for $M$ transmitter $N$ receiver setup operating coherently at each symbol time T, with transmission organized in bursts of $L$ symbols. LST decoding suffers from Multistream Interference (MSI) in which the multiple streams superimpose over one another at the receiver, making the detection difficult. So choice of receiver becomes important to exploit the maximum potential of SM through LST. The three most common receivers are ML, ZF and MMSE. These schemes can be combined with nonlinear detection schemes such as successive cancellation (SUC)

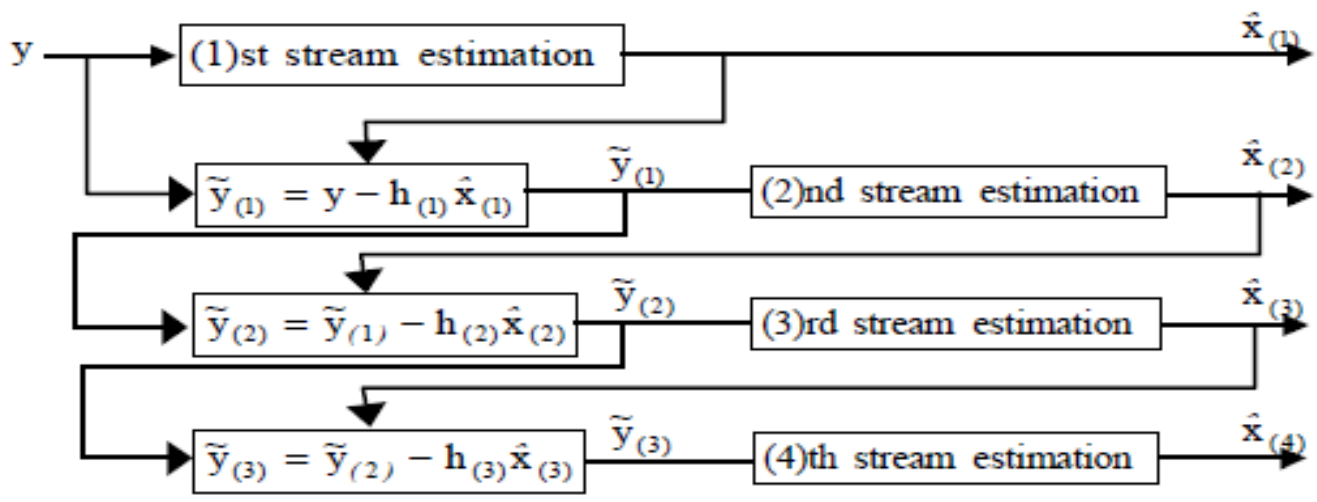

Fig.3 illustration of SUC algorithm

and Optimally Ordered SUC algorithms to enhance the performance. Fig.3 shows SUC algorithm. In SUC-OO, the signal with the strongest SINR is selected for processing at the first stage. This improves the quality of the decision and reduces the chances of error propagation [8]. The various receiver models are described as follows: 


\section{i) ML Receiver:}

This is an optimum receiver, which solves for the estimated symbol vector $\hat{x}$

$$
\hat{x}_{M L}=\arg \min \|y-H x\|^{2}
$$

by searching through all the vector constellation for the most probable transmitted signal vector. The computational complexity is very high as $M$ increases $\left(x^{M}\right)$ but provides full receiver diversity and zero power losses [2].

\section{ii ) ZF V-BLAST Receiver:}

The $\mathrm{ZF}$ receiver is a linear receiver. This technique nullifies the interference by using weight matrix $W_{Z F}$ as follows [3]

$$
\begin{aligned}
& \hat{\mathrm{x}}_{Z F}=W_{Z F} y=\left(\mathrm{H}^{\mathrm{H}} \mathrm{H}\right)^{-1} \mathrm{H} y=x+\tilde{n}_{Z F} \\
& \text { Average noise power is } E\left\{\left\|\tilde{n}_{Z F}\right\|^{2}\right\}=\sum_{i=1}^{M} \frac{\sigma_{n}^{2}}{\sigma_{i}^{2}}
\end{aligned}
$$

This noise in the separated streams are correlated and consequently the SNRs are not independent. The ZF receiver decomposes the link into $M$ parallel streams, each with diversity gain and array gain proportional to $N-M+1$. Hence, it is sub optimum. Recursive SUC-OO Zero Forcing algorithm, which is based on performing the nulling operation using unitary transformations results in a lower complexity and better numerical stability [2]. The recursion determines the elements of the optimal row, which maximizes the Bit Error Rate (BER).

\section{iii ) MMSE V-BLAST Receiver:}

To reduce the impact from the background noise, the MMSE detector employs a linear filter that can take into account the noise. The estimated signal is

$$
\begin{aligned}
& \qquad \hat{\mathrm{x}}_{M M S E}=\mathrm{W}_{M M S E} \mathrm{y}=\left\{\left(\mathrm{H}^{\mathrm{H}} \mathrm{H}+\sigma_{n}^{2} \mathrm{I}\right)^{-1} \mathrm{H}^{\mathrm{H}}\right] \mathrm{y}=\tilde{x}+\tilde{n}_{M M S E} \\
& \text { Average noise power is } E\left\{\mid \tilde{n}_{M M S E} \|^{2}\right\}=\sum_{i=1}^{M} \frac{\sigma_{n}^{2} \sigma_{i}^{2}}{\left(\sigma_{n}^{2}+\sigma_{i}^{2}\right)^{2}}
\end{aligned}
$$

The SUC-OO also can be combined with the MMSE detection method to improve the performance. The MMSE receiver can minimize the overall error caused by noise and mutual interference between the cochannel signals, but this is at the cost of separation quality of the signals. The ZF receiver on the other hand, perfectly separates the cochannels' signals at the cost of noise enhancement. 


\section{RESULTS AND DISCUSSION}

In this section, the performance of various V-BLAST receiver schemes are studied with their BER variation against input signal to noise ratio for different passband modulation techniques. Effects of fading correlation and cross polarization at the antenna on detection are also considered. Study is carried out on effect of antenna spacing and Angle Spread (AS) on spatial correlation. Dropping of Ergodic Capacity for 2x2, 3x3, and 4x4 MIMO system under presence of transmit correlation is simulated. Assumptions made for simulation of $M \mathrm{x} N$ MIMO system are as follows:

- The transmitted signal has a fixed and narrow bandwidth with a flat fading channel model with receiver noise assumed as AWGN.

- The transmitted power is independent of the number of transmit antennas

- The modulation schemes used in the simulation are Mary Phase Shift Keying and Quadrature Amplitude Modulation since these schemes are good indicators of system performance.

Fig.4 indicates the different VBLAST receiver algorithms discussed in this paper for 2x2 MIMO. The ML algorithm gives best BER for given SNR, but the computation complexity increases in the order of $x^{\mathrm{M}}$. Lowest BER is obtained BPSK modulation. The plot also indicates better performance of MMSE over ZF detection algorithm. This is because, the MMSE receiver can minimize the overall error caused by noise and mutual interference between the cochannel signals, but at the cost of separation quality of the signals [5]. For a BER of $10^{-3}$, difference in $E_{b} / N_{o}$ between ML and MMSE-SUC with Optimal Ordering is approximately $1 \mathrm{~dB}$ where as the other schemes require signal strength of approximately $10 \mathrm{~dB}$ more to achieve the same BER.

Fig.5 shows how the cross polarization coupling affects the detector performance. ZF SUC detector is considered with BPSK modulation. For very low

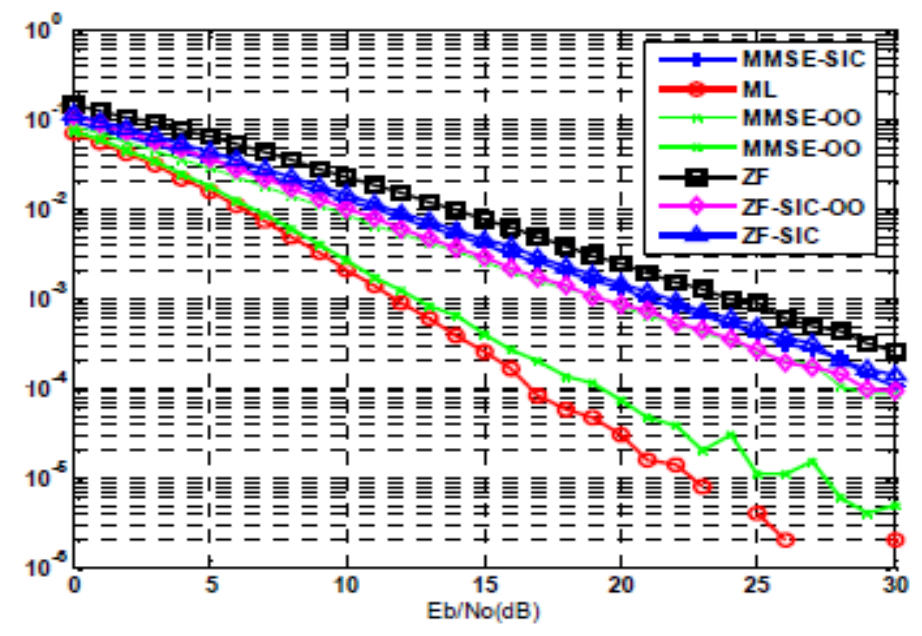

Fig.4 Performance comparison of V-BLAST ML, ZF and MMSE Receivers with and without Successive Cancellation and Optimal Ordering for fading correlation $=1$ and $\alpha=0.5$ 


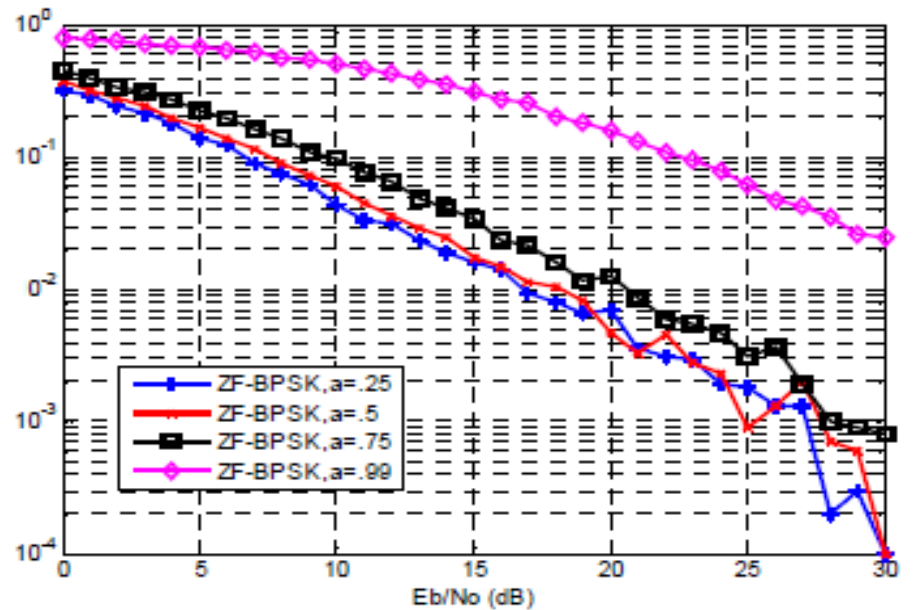

Fig.5 V-BLAST ZF Receiver performance for different cross polarization coefficient values $(\alpha)$

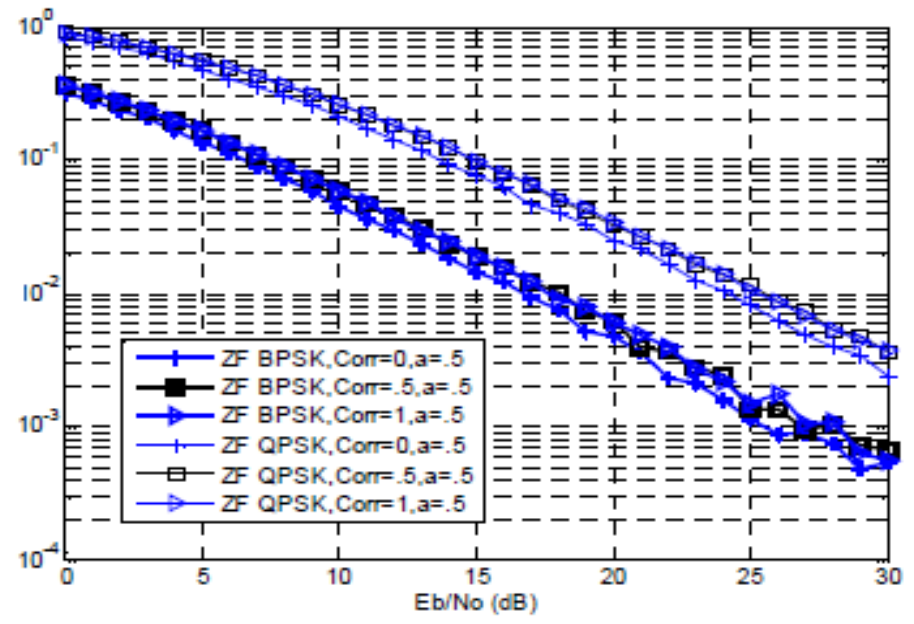

Fig.6 V-BLAST ZF Receiver performance for different correlation coefficient values for BPSK and QPSK modulation, cross polarization coefficient value $(\alpha)=0.5$

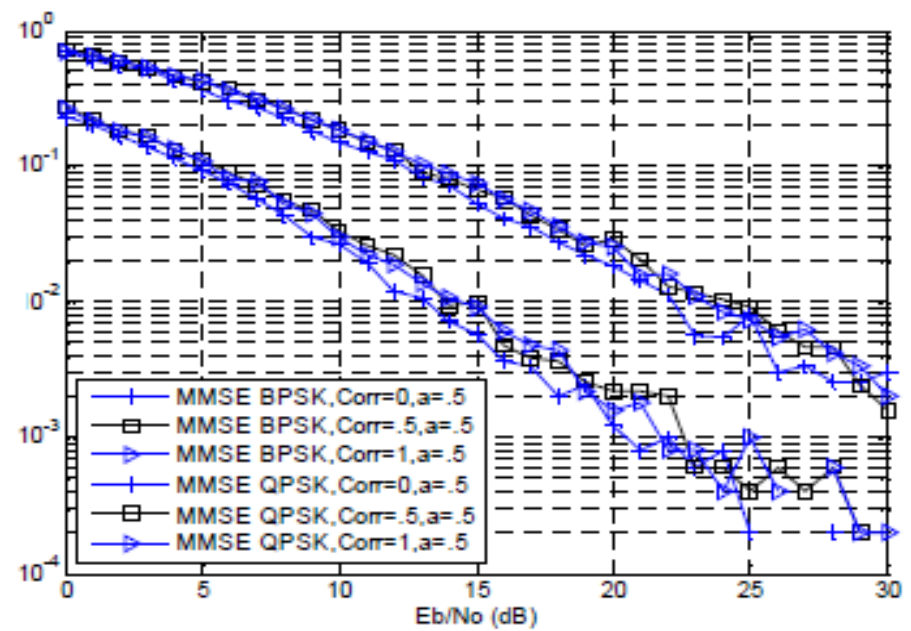

Fig.7 V-BLAST MMSE Receiver performance for different fading correlation values for BPSK and QPSK modulation 


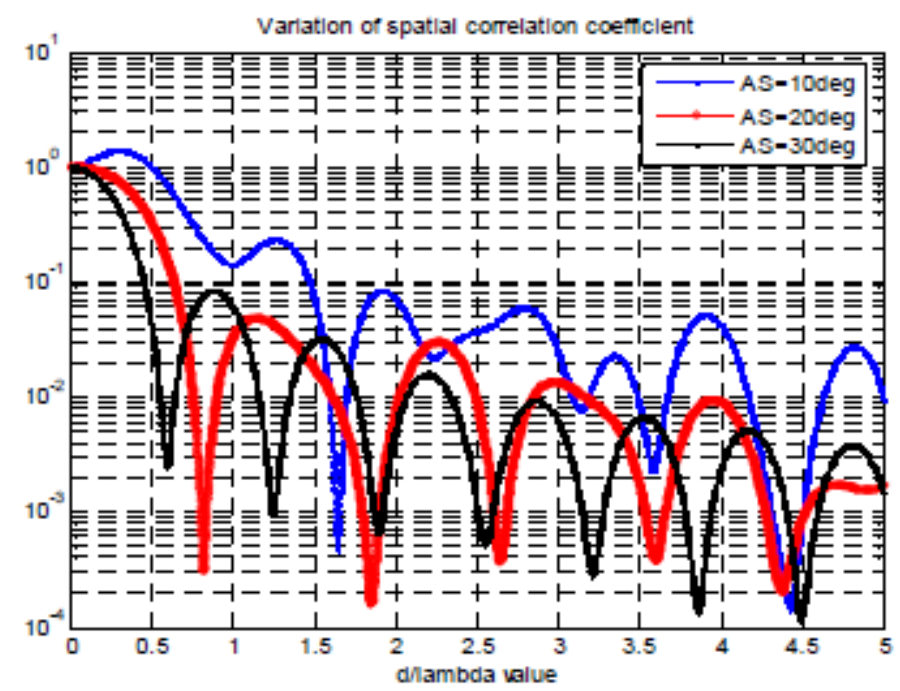

Fig. 8 Variation of Spatial Correlation Coefficient with $\mathrm{d} / \lambda$ for different angular Spread (AS)

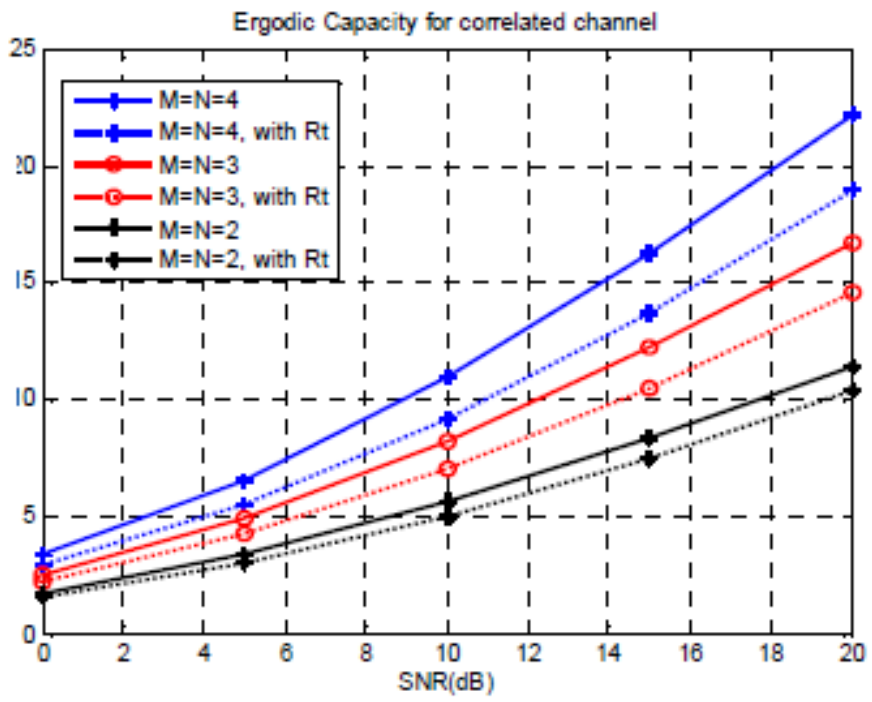

Fig.9 Effect of Correlation on Channel Ergodic Capacity for different MIMO configurations

values of $E_{b} / N_{o}$, the variation of $\alpha$ is not prominent but as $E_{b} / N_{o}$ increases, the effect is more visible. For a BER of $10^{-2}$, the difference is in the order of 2-3dB. $\alpha=1$ implies that the antennas fail to discriminate between each other's signals. This give rise to a downfall in performance as indicated in the result. Numerical results show that for high Cross Polarization Discrimination (XPD) values, single polarized antennas perform better.

Effect of fading correlation coefficient on V-BLAST ZF and MMSE SUC detector is shown in fig.6 and fig.7. Both plots show the general trend for other detectors also. For both BPSK and QPSK, best performance is achieved for zero correlation. If coefficient equals to unity, by (5), the $\mathrm{H}$ matrix will become rank 1 and the channel will reduce to SISO [2]. So lower the correlation, the better. At $B E R=10^{-2}$, the average difference between $E_{b} / N_{o}$ is approximately $2 \mathrm{~dB}$.

Fig.8 shows the variation of spatial correlation coefficient with antenna element spacing $(d / \lambda)$ for different angular spreads. As seen, power distribution becomes broad over large angle as AS 
increases, which decreases the correlation coefficient at the same antenna spacing [8]. The dependency of Ergodic MIMO capacity with correlation between transmit and receive antenna is indicated in Fig.9. For $4 \times 4$ MIMO at SNR of $20 \mathrm{~dB}$, the loss is $3.3 \mathrm{bps} / \mathrm{Hz}$, for $3 \mathrm{x} 3$ system, loss reduces to $2.2 \mathrm{bps} / \mathrm{Hz}$ and for $2 \times 2$ system, it is $1 \mathrm{bps} / \mathrm{Hz}$. At $10 \mathrm{~dB}$ SNR, the corresponding values are approximately 2,1 , and .6bps/Hz respectively.

\section{CONCLUSION}

The impact of receiver fading correlation on the error performance of MIMO systems that employ different V-BLAST receiver algorithms are studied in this paper. It is shown that ML detector gives optimum performance with very high computation complexity.MMSE with Successive Cancellation and Optimum Ordering gives lower BER values, but with a tradeoff in complexity. ZF with Successive Cancellation has lower computational complexity but require more signal power to deliver same error rate. For all these detectors, lower correlation results in better performance. The study is also extended to the effect of cross polarization coupling on the detectors. It is observed that lower coupling value results in lower bit error rate given by the detectors. It is also observed that the Ergodic MIMO capacity reduction is more prominent for larger dimension of MIMO channel matrix.

\section{REFERENCES}

[1] G. J. Foschini and M. J. Gans, "On limits of wireless communications in a fading environment when using multiple antennas," Wireless personal communications, vol. 6, no. 3, 1998, pp. 311-335

[2] Mohinder Jankiraman, "Space-Time Codes and MIMO Systems", Artech House, 2004

[3] Zhongpeng Wang," Grouped Linear ZF Receiver for Multiple Antenna Systems", Proceedings of IEEE International Conference on Communication Software and Networks, 2009, pp 66-68

[4] R.Nabar, H Bolcskei, V Erceg, D Gesbertand A Paulraj, 'Performance of Multiantenna Signaling Techniques in the Presence of Polarization Diversity”, IEEE Trans. Sig. Proc., Vol 50, Issue 10, 2002, pp 2553-2562

[5] Chien-Jen Huang, Chung-Wen Yu, and Hsi-Pin Ma," A Power-Efficient Configurable Low Complexity MIMO Detector" IEEE Transactions on Circuits and Systems I, Vol. 56, No. 2, Feb. 2009, pp 485-496

[6] The Huaping Liu, Yongzhong Song, and Robert C. Qiu," The Impact of Fading Correlation on the Error Performance of MIMO Systems Over Rayleigh Fading Channels", IEEE Trans. on Wireless Communication, Vol.4, No.5, Sept. 2005. pp 2014-2019

[7] Bindu E, B V R Reddy, "Performance analysis of Multiple Detector Algorithms with Optimal Ordering for V-BLAST Architecture in MIMO System", Proceedings of 3rd IEEE IACC, 2013, pp 319-323

[8] Y S Cho, J Kim, W Y Yang, CG Kang, "MIMO-OFDM Wireless Communications with MATLAB”, John Wiley \&Sons, Singapore, 2010

[9] Da-Shan Shiu, Gerard J. Foschini, Michael J. Gans, and Joseph M. Kahn, "Fading Correlation and its Effect on the Capacity of Multielement Antenna Systems", IEEE transactions on Communications, Vol. 48, No. 3, March 2000, pp 502-513 